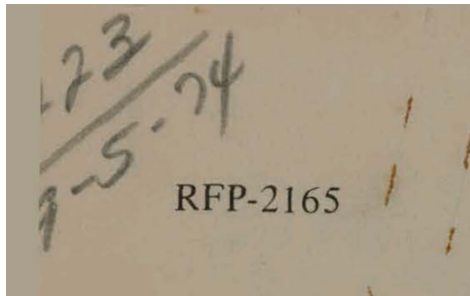

$0 n .928$

RFP-2165

August 5, 1974

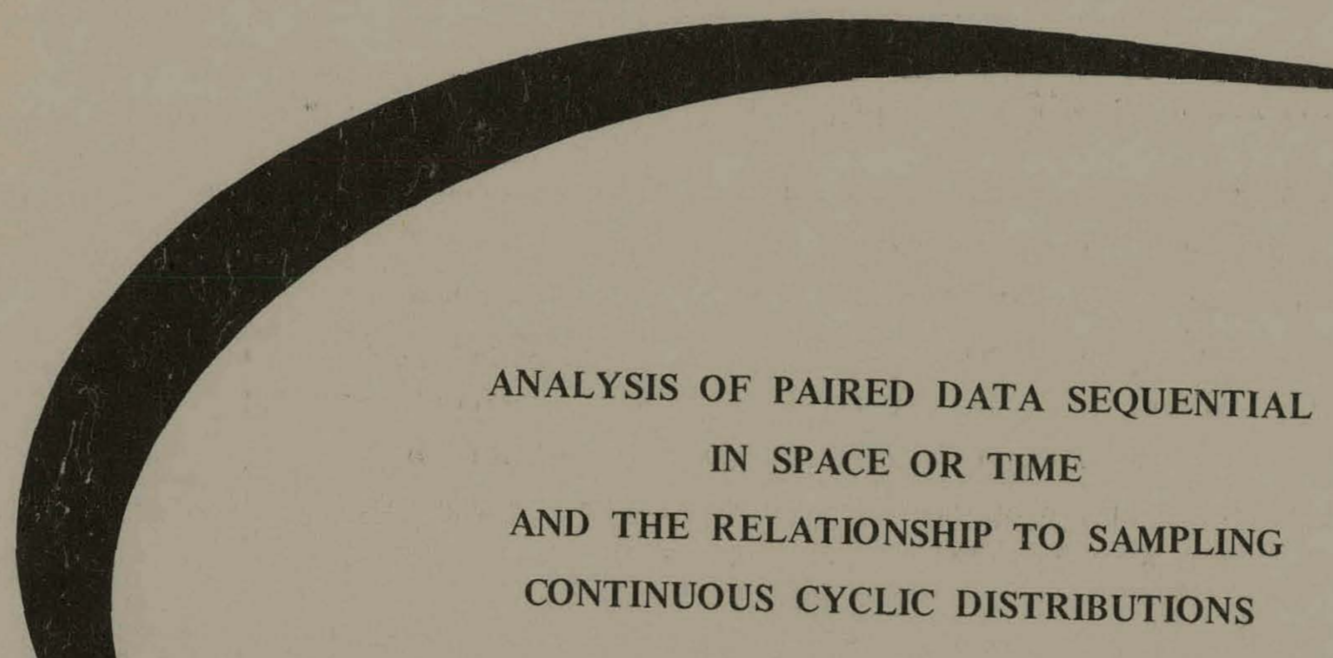

Donald E. Michels

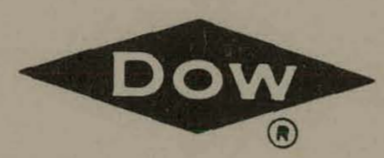

DOW CHEMICAL U.S.A. ROCKY FLATS DIVISION

P. O. BOX 888

GOLDEN, COLORADO 80401

U.S. ATOMIC ENERGY COMMISSION

CONTRACT AT(29-1)-1106 


\section{DISCLAIMER}

This report was prepared as an account of work sponsored by an agency of the United States Government. Neither the United States Government nor any agency Thereof, nor any of their employees, makes any warranty, express or implied, or assumes any legal liability or responsibility for the accuracy, completeness, or usefulness of any information, apparatus, product, or process disclosed, or represents that its use would not infringe privately owned rights. Reference herein to any specific commercial product, process, or service by trade name, trademark, manufacturer, or otherwise does not necessarily constitute or imply its endorsement, recommendation, or favoring by the United States Government or any agency thereof. The views and opinions of authors expressed herein do not necessarily state or reflect those of the United States Government or any agency thereof. 


\section{DISCLAIMER}

Portions of this document may be illegible in electronic image products. Images are produced from the best available original document. 


\section{LEGAL NOTICE}

This report was prepared as an account of work sponsored by the United States Government. Neither the United States nor the United States Atomic Energy Commission, nor any of their employees, nor any of thcir contractors, subcontractors, or their employees, makes any warranty, expressed or implied, or assumes any legal liability or responsibility for the accuracy, completeness or usefulness of any information, apparatus, product or process disclosed, or represents that its use would not infringe privately owned rights.

Printed in the United States of America

Available from the

National Technical Information Service

U. S. Department of Commerce

Springfield, Virginia 22151

Price: Printed Copy $\$ 4.00$ Microfiche $\$ 1.45$ 


\title{
ANALYSIS OF PAIRED DATA SEQUENTIAL IN SPACE OR TIME \\ AND THE RELATIONSHIP TO SAMPLING \\ CONTINUOUS CYCLIC DISTRIBUTIONS
}

\author{
Donald E. Michels
}

Product and Health Physics Research

HEALTH PHYSICS RESEARCH GROUP

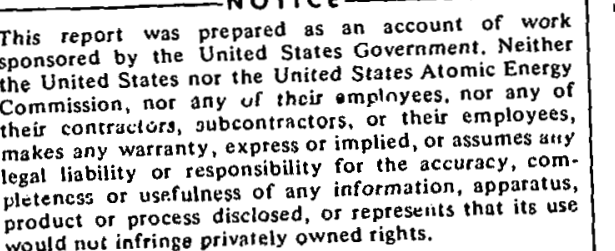

product or process disclosed, or seprese
would nut infringe privately owned rights.

\section{SUBJECT DESCRIPTORS}

DOW CHEMICAL U.S.A.

ROCKY FLATS DIVISION

P. O. BOX 888

GOLDEN, COLORADO 80401

Prepared under Contract AT(29.1)-1106

for the

Albuquerque Operations Office

$U$. S. Atomic Energy Commission
Data Analysis

Sampling 
RFP-2165 


\section{CONTENTS}

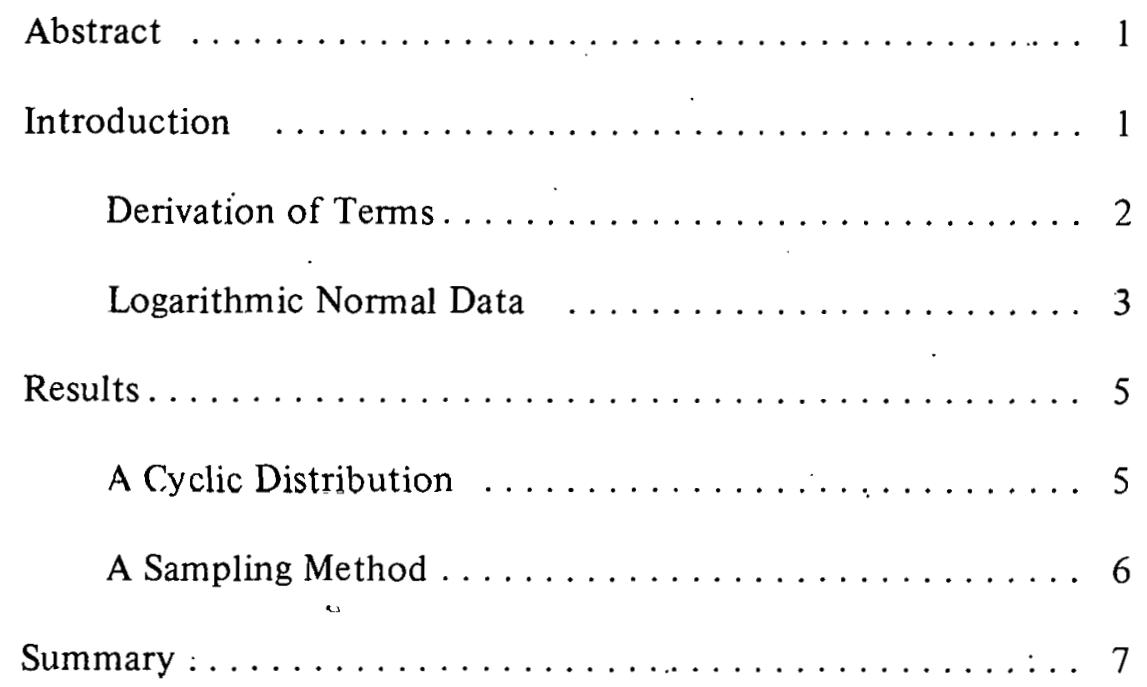




\title{
ANALYSIS OF PAIRED DATA SEQUENTIAL IN SPACE OR TIME AND THE RELATIONSHIP TO SAMPLING CONTINUOUS CYCLIC DISTRIBUTIONS
}

\author{
Donald E. Michels
}

\begin{abstract}
Three standard deviations are used to determine what fraction of available information may have been obtained from a set of samples. Discrete data are arranged sequentially in either space or time, and the subject is presumed to exist as a continuous cyclic variable with a continuous trend. The difference between variance for samples of a set and variance for multiple analyses of a single sample define the maximum amount of information obtainable from the set. However, for pairs of samples adjacent in the sequence, variance has an intermediate value. The resolution of the set of samples depends on where the variance for adjacent pairs fits in the range bounded by the other variances. The resolution actually obtained can then be compared with a desired resolution in order to decide whether resampling is required, worth the cost, or whether a new analytical method is needed instead.
\end{abstract}

A procedure for taking composite samples can be based on the cycle length of the continuous variable. Cycle length may be indicated by the standard deviation for adjacent pairs. A worked

NOTE: The author is currently with Environmental Services, Acrojet Nuclear Company, Idaho Falls, Idaho 83401. example problem involves cesium-137 fallout in a series of soil samples.

\section{INTRODUCTION}

All samples of surface soil " contain radioactive debris from fallout; the amount, however, varies from place to place. I'hus, the levels of fallout form a continuous distribution across space. ${ }^{2}$ The taking of discrete samples from a continuous distribution, as in sampling soil for fallout, may be complicated by how the sample spacing matches . cyclic $^{3}$ variations in concentrations of the material under study. The variations may originate either from random or from systematic events.

The scale of cyclicity will generally be unknown at the time the first samples are taken, hence, the variability among the samples involves questions about how analytically determined values yield information about the cyclicity. Figure 1 shows

\footnotetext{
'Soil taken from sites which were exposed to fallout events.

${ }^{2}$ The distribution with time sometimes is studied and the concepts here apply to distributions over time as well.

${ }^{3} \mathrm{Cyclic}$ is used in the sense of regions of high values separated by regions of low values with no specification about regularity.
}

FIGURE 1. Schematic of How Discrete Samples (1) May Represent a Continuing Cyclic Distribution (2).

Legend

1. Error bars on data points indicate uncertainty inherent to sampling and analysis.

2. Continuous line represents the absolute value of the concentration apart from any experimental difficulties in measurement.

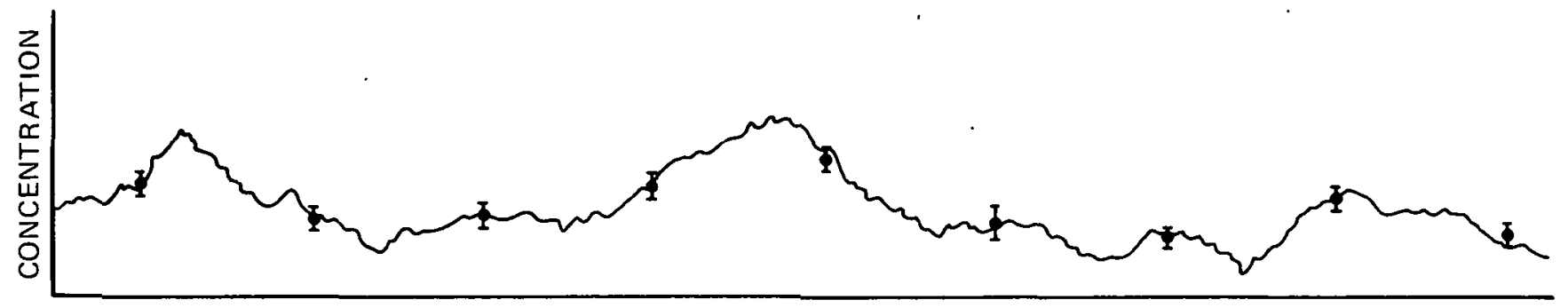

OISTANCE 
schematically how discrete samples may be related to the absolute values of a continuous distribution. Cyclicity that is small compared to the spacing of samples will appear indistinguishable from analy tical noise whereas the longer wavelength variations may be well resolved. Because the cyclic scale(s) are unknown, initially, the first choice sample,spacing must be made arbitrarily. When data become available one may ask, has important variability been missed because of too coarse a sample spacing or, were more samples taken than necessary to just resolve the interesting variability? Answers to either of those questions can be quantified from the first set of samples and such quantification is part of the subject of this report.

A related concern is the taking of composite samples that are intended to be representative of a site or a region. The elements of an ideal composite should balance statistical biases. This is to say that the logic in making a composite involves the presumption that a single element of the composite is not reliable, but that multiple elements might be reliable. Usually, elements are taken broadly so that real variations between them will be randomized. The practical question involves the second feature of this report, quantifying randomness among the elements of a composite sample.

Besides pertinence to sampling soil, similar considerations may be useful for biological data. For example, the level of plutonium in urine excreted during a year's time by a person is a continuous cyclic variable with a continuous trend. Choice of sampling frequency (in time) and interpretations of data should recognize the cyclicity even when the trend is the major concern.

In quantifying how data from spaced samples fit cyclicity in the distribution of the subject, the classical standard deviation among data in a set is compared both to the analytical standard deviation and to the deviation between data that are adjacent in the sequence.

\section{Derivation of Terms:}

The equation for classical standard deviation (SD) is:

$$
\mathrm{SD}=\left[\frac{\sum_{\mathrm{i}=1}^{N}\left(\mathrm{x}_{\mathrm{i}}-\overline{\mathrm{x}}\right)^{2}}{\mathrm{~N}-1}\right]^{1 / 2}
$$

Equation 2 may be rewritten for the case of only two samples:

$$
\mathrm{SD}_{2}=\frac{1}{\sqrt{2}}\left|\mathrm{x}_{\mathrm{i}}-\mathrm{x}_{\mathrm{j}}\right|
$$

For a series of $\mathrm{N}$ data, $(\mathrm{N}-1)$ values may be ohtained from the $\mathrm{N}-1$ adjacent pairs of data. The result is defined as standard deviation for adjacent pairs (SDAP):

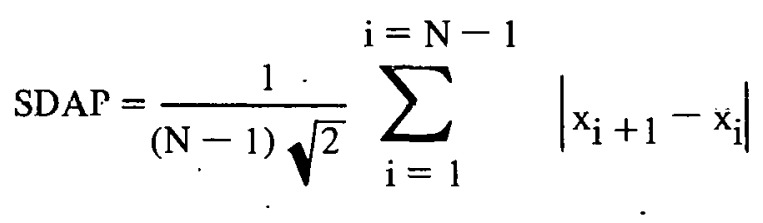

If the data arc random, then the average SDAP will bc statistically identical, although not numerically identical, with the classical value of SD given by Equation 1. However, if the data are correlated, in the sensc that adjacent values come from the same limb of a continuous cyclic function, then the SD $\Lambda P$ will be statistically smaller than the SD.

Analytical precision provides a lower liniting value for SDAP; adjacent samples cannot be more similar than replicates of a single sample which provide the estimate of analytical precision (AR). ${ }^{4}$

Value of SDAP may be larger than SD only (yet not always) when the sampling frequency is large compared to the half-cycle.length of the continuous variable under study. In such cases, only an average value may be given for the continuous variable-there would be no resolution of cycle length.

Whether the SD is significantly different from the SDAP can be determined by the classical F Test:

\footnotetext{
${ }^{4}$ Analy tical precision is the standard deviation for replicate aliquots of a single sample.
} 


$$
\mathrm{F}=\frac{(\mathrm{SD})^{2}}{(\mathrm{SDAP})^{2}}
$$

In Equation 4, $\mathrm{N}-1$ degrees of freedom are involved in both numerator and demoninator. Significance is determined from the usual statistical table.

If the F Test does not show significance, then the discrete values should be used only to estimate averages or trends. Resampling at a shorter interval should be considered, or for soils, fill-in samples could be obtained. If the F Test shows significance then the two values, SD and SDAP, may be used as components of variance, along with analytical precision (AR), to quantify the amount of information contained among the discrete values.

In concept, the difference between SD and SDAP represents information obtained; the difference between SDAP and analytical precision (AR) represents information lost due to coarseness of the sampling frequency. The three values (SD, SDAP, and AR) are not directly additive; however, the squares of the three values are additive as components of variance. Thus, the amount of information lost is indicated by Equation 5; the amount gained by Equation 6 .

$$
\begin{aligned}
& \text { Information Lost } \\
& \text { is Proportional to [(SDAP } \left.)^{2}-(A R)^{2}\right]
\end{aligned}
$$

The bracketed term in Equation 5 represents variability between data points, a variability that cannot be resolved further with the available data. The corresponding bracketed term in Equation 6 represents the amount of variability in the set of data which can be further resolved by additional manipulations:

$$
\begin{aligned}
& \text { Information Gained } \\
& \text { is Proportional to }\left[(\mathrm{SD})^{2}-(\mathrm{SDAP})^{2}\right]
\end{aligned}
$$

The maximum information possible to gain from a set of data is given in Equation 7:

$$
\begin{aligned}
& \text { Maximum Information } \\
& \text { is Proportional to [(SD })^{2}-(A R)^{2} \text { ] }
\end{aligned}
$$

This condition occurs in practice when the SDAP equals (statistically) the AR.
A parameter, I, can be computed indicating the amount of information actually resolved by the samples as a fraction of the information that would have been gained if a continuous set of samples had been taken instead:

$$
I=\frac{(\mathrm{SD})^{2}-(\mathrm{SDAP})^{2}}{(\mathrm{SD})^{2}-(\mathrm{AR})^{2}}
$$

When I values exceed about 0.6 , the data are sufficiently dense to resolve some cyclic variability in a continuous distribution. These cases justify a regression curve fit to a cyclic model, either empirical or theoretical. For smaller values of I, the data should be used only for indicating trends of larger scale than the sampling spacing. One should be aware that unresolved cyclic variability exists within the trend.

In a sampling program, a choice may be needed between changing the frequency of sampling or changing the inherent precision of the analytical results. Equations 5, 6, and 7 can be used to help decide which change would be most cost effective. For example, if values from Equation 5 exceed those from Equation 6, then more samples are needed at closer intervals or, depending on circumstances, a less precise analy tical method might be acceptable. Whereas a small value from Equation 7 suggests that an improved analy tical method is needed. In some cases, the AR may be improved by increasing either the sample size or the number of replicate analyses made for each sample point, rather than developing a new technique of analysis.

\section{Logarithmic Normal Data:}

Statistical tests are readily available for data that conform to Gaussian distributions. However, since many environmental data are not Gaussian, 5, 6, 7

\footnotetext{
${ }^{5}$ R. J. Larsen. A Mathematical Model for Relating Air Quality Measurements to Air Quality Standards. U.S. Environmental Protection Agency, U.S. Government Printing Office, Washington, D. C. 1971 .

${ }^{6}$ A. R. Miesch. Methods of Computation for Estimating Geochemical Abundance. Professional Paper 574-B. U.S.Geological Survey, U.S. Government Printing Office, Washington, D. C. 1967.

${ }^{7}$ D. A. Rodionov. Distribution Functions of the Element and Mineral Contents of Igneous Rocks. Consultants Bureau Transactions, New York. 1965.
} 


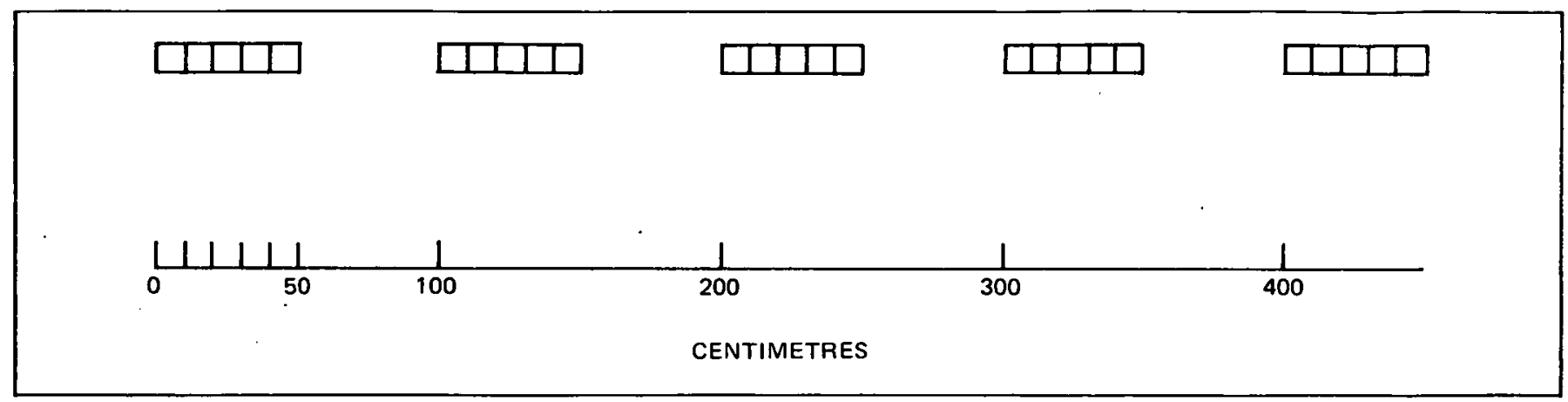

FIGURE 2. Layout of Sample Sites.

some adjustment is required for either the data or the statistical tests. Usually, data transformation is easier. A logarithmic transformation has many utilities and is achieved by simply using the logarithms of data instead of the data themselves in all equations derived previously.

Similarly, all statistical tests and conclusions are based on computations involving logarithms rather than raw data. Conventional units can be restored to the statistical quantities by taking anti-logarithms as a subsequent step after any manipulation. The correlations between Gaussian and logarithmic normal statistical parameters are given by Kalinske. ${ }^{8}$ Logarithmic normal statistics may he preferred when the coefficient of variation exceeds 0.3."

When anti-logarithms are taken of the statistical quantities, the results are referred to as geometric quantities; for example, geometric standard deviation (GSD) and geometric average (GA).

$$
\begin{gathered}
\mathrm{GA}=\exp \frac{1}{\dot{\mathrm{N}}} \sum_{i=1}^{N}\left(\ln x_{i}\right) \\
=\exp (\overline{\ln \mathrm{x}}) \\
\neq \exp (\ln \overline{\mathrm{x}})
\end{gathered}
$$

\footnotetext{
${ }^{8}$ A. A. Kalinske. "On the Logarithmic-Probability Law." Transactions of the American Geophysical Union 27:709. 1946.

${ }^{9} \mathrm{G}$. S. Koch and R. F. Link. "The Coefficient of Variation, A Guide to the Sampling of Ore Deposits." Economic Geology 66:293. 1971.
}

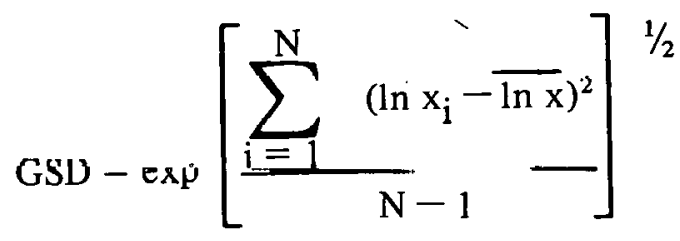

If logarithms were used for $S \mathrm{D}, \mathrm{SDAP}$, and AR, then their geometric counterparts are:

$$
\begin{aligned}
& \text { GSD }=\exp (\mathrm{SD}) \\
& \text { GSDAP }=\exp (\mathrm{SDAP}) \\
& \mathrm{GAR}=\exp (\mathrm{AR})
\end{aligned}
$$

Considerable convenience may be gained by these geometric expressions because their application relates closely to concepts about coefficient of variation and percent variation. A GSD of 1.48 means that a value 1 -sigma removed from the GA is $(1.48)^{1}$ times larger (or smaller than) the GA; a 1-sigma variation of 48 percent. A value 2 -sigma removed from the GA is $(1.48)^{2}$ times larger (or smaller) than the GA.

When Gaussian data are treated log-normally, no significant changes occur from the outcome of statistical tests nor in the subsequent conclusions. However, the converse of treating log-normal data as if it were Gaussian can lead to improper results. Levels of significance are inflated inappropriately, and a considerable risk involves rejecting a true null hypothesis. 
Often data of two origins are to be compared, for example the levels of fallout from cesium (Cs) and plutonium $(\mathrm{Pu})$. The $\mathrm{Cs}$ data are fairly represented by Gaussian statistics, but $\mathrm{Pu}$ data are better treated log-normally. In such cases, comparisons between the two materials are better if the statistics have the same basis. Thus, a necessary log-normal treatment of $\mathrm{Pu}$ data makes advisable a log-normal treatment of Cs data even though a Gaussian treatment would be appropriate for Cs alone.

\section{RESULTS}

\section{A Cyclic Distribution:}

An example of applying these concepts to fallout data comes from a series of 25 ten-centimetre $(\mathrm{cm})$ square soil samples taken along a 5-metre $(\mathrm{m})$ line. Each sample was $5 \mathrm{~cm}$ deep and included vegetation. Samples are from a gently sloping well-vegetated shelf of a valley at the Rocky Flats site. Figure 2 shows the layout, and Table I shows data for cesium-137.

A log-normal statistical model is used, though not required here. The layout provides for computing 3 values for GSDAP, one for each of 3 sample spacings, $0.1,1$, and 2 metres respectively. Each can be compared independently with GSD and GAR as shown in Table II. The GAR was determined by returning a counted aliquot of soil to the remainder of the sample, realiquotting, and recounting. Eleven such pairs of data were used to estimate the GAR, the number of degrees of freedom (df) reflected in Table II.

The GAR is statistically the same as the GSDAP $(0.1 \mathrm{~m})$. Since samples $10 \mathrm{~cm}$ across cannot be taken closer than $10 \mathrm{~cm}$ center-to-center, the GSDAP $(0.1 \mathrm{~m})$ is a practical substitute for an estimate of analytical precision. Both the GSDAP $(0.1 \mathrm{~m})$ and GSDAP $(1 \mathrm{~m})$ are significantly smaller than the GSD. This outcome indicates real variability along the 5-metre traverse and conforms to the model of a cyclic continuous function for Cs-137 levels. Nonsignificance betwecn GSD
TABLE I. Cesium 137 in Samples from a Linear Array.

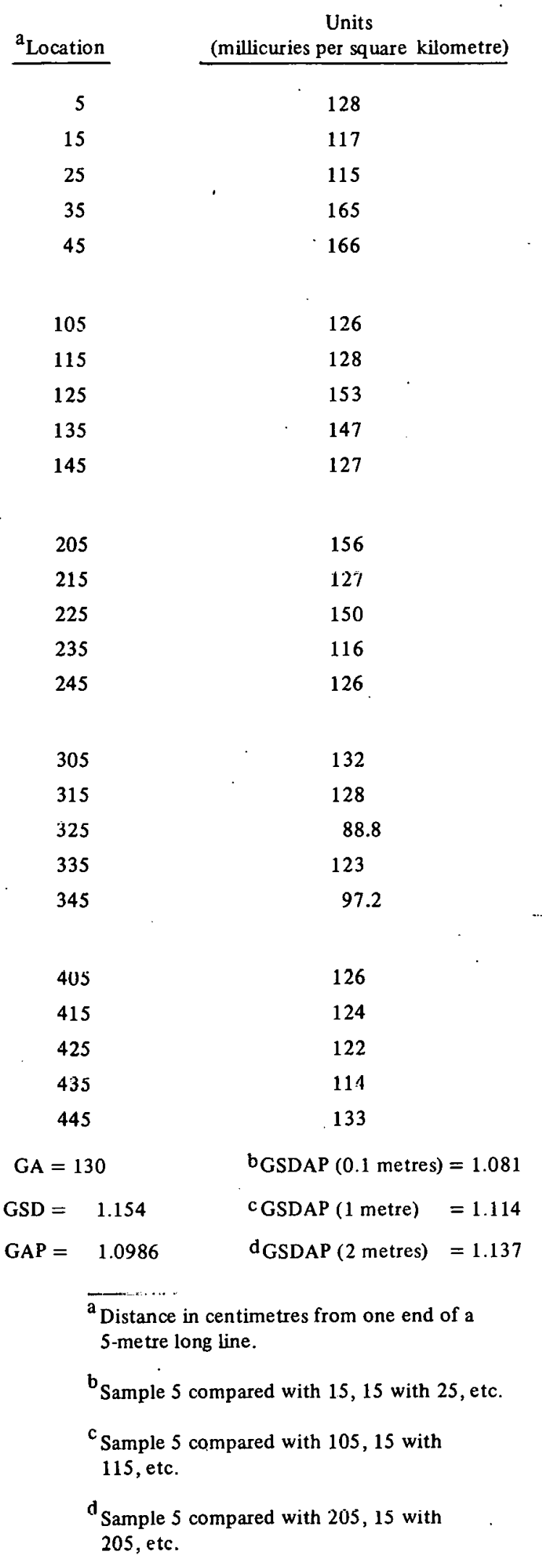


TABLE II. Significance of Selected Variabilities.

\begin{tabular}{|c|c|c|c|}
\hline \multirow[b]{2}{*}{$\log G S D$} & F Ratio & $\begin{array}{l}\text { Degrees of } \\
\text { Freedom }\end{array}$ & $\begin{array}{c}\text { Significance } \\
\text { Level }\end{array}$ \\
\hline & \multirow{2}{*}{3.38} & 24 & \multirow{2}{*}{0.996} \\
\hline $\log \operatorname{GSDAP}(0.1$ metre) & & $\overline{20}$ & \\
\hline $\log$ GSD & \multirow{2}{*}{1.76} & 24 & \multirow{2}{*}{0.90} \\
\hline log GSDAP (1 metre) & & 20 & \\
\hline $\log$ GSD & \multirow{2}{*}{1.24} & 24 & \multirow{2}{*}{0.65} \\
\hline log GSDAP ( 2 metres) & & 15 & \\
\hline log GSDAP (0.1 metre) & \multirow{2}{*}{0.686} & 20 & \multirow{2}{*}{0.22} \\
\hline $\log$ GAR & & 11 & \\
\hline $\log$ GSDAP ( 1 metre) & \multirow{2}{*}{1.32} & 20 & \multirow{2}{*}{0.66} \\
\hline $\log$ GAR & & 11 & \\
\hline $\log$ GSDAP ( 2 metres) & \multirow{2}{*}{1.86} & 15 & \multirow{2}{*}{0.83} \\
\hline $\log$ GAR & & 11 & \\
\hline $\begin{aligned} \mathrm{GSD} & =\mathrm{Gec} \\
\mathrm{GSDAP} & =\mathrm{Ge} \\
\mathrm{GAR} & =\mathrm{Ge}\end{aligned}$ & $\begin{array}{l}\text { netric Star } \\
\text { hetric Star } \\
\text { djacent Pa } \\
\text { hetric Ana }\end{array}$ & $\begin{array}{l}\text { ard Devlation. } \\
\text { ard Deviation } \\
\text { tical Precision. }\end{array}$ & \\
\hline
\end{tabular}

and GSDAP ( $2 \mathrm{~m}$ ) indicates that samples 2 metres apart are not (usually) on the same limb or cycle.

The relative merits of sampling at different spacings can be determined from Equations 6 and 7 and the values listed in Table I. Maximum information obtainable from the analy tical precision is proportional to Equation 7 and thus Equation 14 is:

$$
\begin{aligned}
& \left(\ln ^{2} \mathrm{GSD}\right)-\left(\ln ^{2} \mathrm{GAR}\right) \\
& =0.02052-0.00884=0.0116 \%
\end{aligned}
$$

Information obtained with the 2-metre separation of samples is proportional to:

$$
\left(\ln ^{2} \mathrm{GSD}\right)-\left[\ln ^{2} \operatorname{GSDAP}(2 \mathrm{~m})\right]=0.00470
$$

The ratio of Equation 14 to Equation 15 indicates the fraction of information recovered by samples separated 2 metres apart, 0.40 .

Similarly, the $1-\mathrm{m}$ separation could recover 0.57 of the information. The $0.1-\mathrm{m}$ separation recovers all the information obtainable from analyses of existing GAR.

When the sampling objective concerns description of the continuous variable function, quality of sampling can be judged by the preceding process regardless of the scale of sampling involved. Cost effectiveness can also be estimated. In the example above, doubling the number of samples by halving their separation ${ }^{10}$ appears to increase the gained information by only 42 percent $(0.57-0.40 / 0.40)$, but will nearly double the cost of analysis. Whether the increase in gained information will be cost effective depends on other criteria.

In this example, the extra cost would be justified since the 2-m separation fails to demonstrate the cyclic distribution function that is apparent with the 1-m separation (Table II).

\section{A Sampling Method:}

Not all sampling is done to characterize an underlying distribution function. In at least one case, sampling must be done to hide the underlying distribution. For example, a site several metres square might be selected for fallout sampling because it was thought to be representative of a larger area, perhaps thousands of metres across.

A practical soil-sampling procedure involves the taking of several cores which are combined. The area of the composite sample is the sum of the areas of the separate cores. For a chosen accuracy in representing the site, one must choose a minimum number of cores and specify a juxtaposition that conforms to randomization.

The continuous distribution function of fallout extends laterally, as well as longitudinally, and

\footnotetext{
${ }^{10}$ For areal rather than linear arrays, halving the separation causes a four-fold increase in number of samples.
} 
the separate cores should be spaced far enough apart so that no two lie on the same limb of a cycle. Also, the spacing should not be regular because of the (unlikely) possibility that the underlying cycle length of the distribution function might be some integer multiple of a selected sample spacing. The outcome of Table II shows that a 1-metre separation of cores could be unsatisfactory (at the example site), because adjacent cores are highly correlated, not random.

If adjacent cores are highly correlated, by virtue of their proximity, then one is (partly) redundant. Such a redundancy makes the composite sample unnecessarily large. For example, a composite of 5 widely spaced cores will be only half as large as a composite of 5 pairs of cores. Yet when each member of a pair is taken close to its mate, either composite will represent the site with nearly identical accuracy.

Achieving representativeness by compositing becomes more important as the variability of the distribution function increases. In the example, 1 -sigma variability for 100 -square $\mathrm{cm}$ samples is near 15 percent of the central value $(\mathrm{GSD}=1.154$ ), yet one may wish to sample the site to obtain precision of \pm 5 percent (GSD near 1.05).

Multiple samples of 100-square $\mathrm{cm}$ each could be composited to achieve the higher precision. How many are needed can be calculated by noting that the precisions of single samples and composites are related by the square root of the number of samples in the composite as shown in Equation 16.

$$
\begin{aligned}
\ln 1.05 & =\frac{\ln 1.154}{\sqrt{n}} \\
n & =8.6 \simeq 9
\end{aligned}
$$

Thus, 9 samples of 100 -square $\mathrm{cm}$ each are needed to sample the site to a precision of \pm 5 percent. But the distribution of these 9 samples also is important. A one-metre separation is lou cluse, as indicated by the high correlation (nonrandom) shown in Table II. A 2-metre minimum separation would be adequate.

A preferred sampling method based on the concepts and data above would have the parts of the composite randomly spaced throughout the sample site with the stipulation that no 2 are closer than 2 metres. Whether this method is adequate or overly restrictive at other places depends on the scales of variability which exist there.

\section{SUMMARY}

A series of 25 soil samples taken along a line shows levels of Cs-137 that conform to a continuous cyclic distribution function for fallout. Samples close to one another tended to be similar in Cs content, the contents being more similar as the spacing was reduced. Similarity in contents versus proximity indicated that the half-cycle length of the distribution function was greater than 1 metre, though possibly less than 2 metres.

The relationship between proximity and similarity was quantified by three statistical quantities, classical variance, $(\mathrm{SD})^{2}$, variance between adjacent pairs of samples, and variance of replicate aliquots. The amount of information actually obtained from a set of samples can be compared to the maximum obtainable, permitting judgments about whether resampling or changes in sample frequency would be worthwhile in terms of information gained versus cost.

A sampling method was described based on the standard deviation of single samples and the cycle dimensions of the distribution function. The method specifies a minimum separation between samples and tells how many samples of a given size must be composited to achieve a desired level of accuracy in sampling.

A worked example assumes logarithmic normal distributions which are recommended for most environmental data. 
RFP-2165 